\title{
Determinação dos valores normais do eletrorretinograma de campo total em cães da raça Poodle portadores de catarata de acordo com a faixa etária
}

\author{
Determination of normal values of full-field electroretinogram in Poodle dogs with cataract \\ according to age
}

\author{
Angélica de Mendonça Vaz Safatle ${ }^{\mathrm{I} *}$ Ricardo Lisak $^{\mathrm{I}}$ Denise Aya Otsuki ${ }^{\mathrm{I}}$ Débora Gomes $^{\mathrm{I}}$
}

RESUMO

Eletrorretinograma (ERG) é um meio diagnóstico objetivo, não invasivo e capaz de avaliar a função retiniana, detectando precocemente, em diversas espécies, lesões nas camadas mais externas. Tem como uma das principais indicações a avaliação pré-cirúrgica em pacientes portadores de catarata. A determinação dos valores normais do ERG é indispensável quando se objetiva, pela mensuração dos valores, o auxílio no diagnóstico das degenerações retinianas, mesmo quando apresentam opacidade de meios (catarata). Este estudo teve como objetivo padronizar os valores das respostas do eletrorretinograma em cães da raça Poodle portadores de catarata madura ou hipermadura, submetidos ao mesmo protocolo de sedação, adaptação ao escuro e eletrodo, utilizando aparelho portátil BPM 200. Em todos os animais, o exame oftalmológico precedeu o ERG. Os animais foram separados em quatro grupos de acordo com a faixa etária: um a três anos (GI), quatro a sete anos (GII), oito a 11 anos (GIII) e 12 anos ou mais (GIV). Foram mensuradas a amplitude pico a pico (pico da onda-a até o pico da onda-b) e o tempo de culminação da onda-b na resposta de bastonetes, máxima resposta e resposta de cones. Cães com respostas atenuadas ou extintas foram excluídos deste trabalho. Observou-se diminuição das amplitudes das três respostas quando se comparou GI com os demais grupos. Houve aumento do tempo de culminação da onda-b na comparação da resposta de bastonetes de GI com os outros grupos. O tempo de culminação da onda-b na máxima resposta e resposta de cones mantevese praticamente inalterado nos quatro grupos testados. Concluiu-se que houve alteração dependente de idade nos parâmetros eletrorretinográficos, com diminuição da amplitude na resposta de bastonetes, máxima resposta e resposta de cones, enquanto que, no tempo de culminação da onda-b, apenas a resposta de bastonetes foi estatisticamente significante nos animais submetidos ao mesmo protocolo na execução do ERG.

Palavras-chave: cães, Poodle, catarata, eletrorretinograma.

\begin{abstract}
Electroretinogram (ERG) is an objective and noninvasive diagnostic tool for the evaluation of retinal function, capable to detect early, lesions of the outermost layers in several species of animals. One of its main indications is to evaluate pre-surgical patients for cataract surgery. The determination of normal ERG values is essential if the aim is, by measuring the values; aid the diagnosis of retinal degeneration, despite of the presence of media opacity (cataract). The objective of this study was to standardize the values of electroretinogram responses in Poodle dogs with mature or hypermature cataract, submitted to the same sedation and dark adaptation protocol, using a portable electrodiagnostic system, BPM 200. In all animals, the ophthalmologic examination preceded ERG. Animals were divided into four groups according to age: 1 to 3 years (GI), 4 to 7 years (GII), 8 to 11 years (GIII) and 12 or more years (GIV). Peak-to-peak amplitude (peak of a-wave to peak of b-wave) and $b$-wave implicit time were measured in rod response and in maximal response and cone response. Dogs with decreased or absent responses were excluded from the study. GI presented decrease in amplitudes of the three responses and increase in b-wave implicit time of rod response when compared with the other groups. B-wave implicit times in maximal response and cone response were practically unaltered in all four groups. It was concluded that there was agedependent changes in electroretinographic parameters, with decreased amplitudes in rod response, maximal response and cone response. Delay in b-wave implicit time was observed in rod response and unchanged in the two others tested responses of animals subjected to the same ERG protocol.
\end{abstract}

Key words: $d o g$, Poodle, cataract, electroretinogram.

\section{INTRODUÇÃO}

Eletrorretinograma (ERG) é a onda polifásica retiniana obtida em resposta ao estímulo luminoso

IClínica Vetmasters, 01415-004, São Paulo, SP, Brasil. E-mail:angsaf@usp.br.*Autor para correspondência. 
(GONÇALVES et al., 2000). Essa resposta é complexa, pois é o resultado do conjunto da atividade de milhões de células retinianas (BIRCH, 1989; BIRCH \& ANDERSON, 1992; DANTAS, 1995; YANASE \& OGAWA, 1997). A sua importância se dá por tratar-se de meio diagnóstico objetivo (TZEKOV \& ARDEN, 1999), não invasivo (SAFATLE et al., 2005) e capaz de avaliar a função retiniana, detectando precocemente lesões nas suas camadas mais externas (MARMOR \& ZRENNER, 1998; KOMAROMY et al., 1998a). Por ser o método de diagnóstico mais preciso para se avaliar a função da retina (KOMAROMY et al., 1998a), tem inúmeras aplicações, tanto na Medicina Humana, como na Medicina Veterinária.

Em cães, as indicações mais comuns para ERG são: avaliação pré-cirúrgica de pacientes com catarata, caracterização de distúrbios que causam cegueira como glaucoma, acromatopsia (KOMAROMY et al., 1998b; RUBIN, 1971; SIMS, 1999), displasia retiniana, retinopatias degenerativas (OFRI et al., 1993; SAFATLE et al., 2005), hipoplasia de nervo óptico, degeneração retiniana repentina adquirida e lipofuccinose ceroide neuronal (NARFSTRÖM et al., 2007). As avaliações eletrorretinográficas em cães propiciam também um importante modelo para o estudo da distrofia retiniana que acomete o homem (PETERSEN-JONES et al., 2006).

A catarata, que representa a principal causa de cegueira tratável nos cães, é uma doença frequente na raça Poodle em diferentes idades (ADKINS \& HENDRIX, 2005; GELATT \& MACKAY, 2005), impossibilitando a oftalmoscopia e detecção de qualquer anormalidade no segmento posterior. O ERG tem então importância ímpar na avaliação da função retiniana nesses animais. Estudos em cães demonstram que, quanto mais jovem, maiores são as amplitudes e, quanto mais velho, menores são as amplitudes das respostas, semelhante ao que ocorre no ERG humano (NARFSTRÖM et al., 2002).

Em virtude da presença de muitas variáveis, como método de registro, estímulo, adaptação ao escuro, protocolo de sedação ou anestesia que podem alterar os resultados do ERG, é indicado que cada laboratório tenha seus parâmetros de normalidade (MENTZER et al., 2006; NORMAN et al., 2008). Objetivou-se, com este estudo, padronizar os valores de normalidade da resposta de bastonetes, máxima resposta e resposta de cones em cães da raça Poodle portadores de catarata madura ou hipermadura, de acordo com a faixa etária, utilizando aparelho portátil BPM 200.

\section{MATERIAL E MÉTODOS}

ERG de campo total foi realizado em 257 olhos de cães da raça Poodle (151 animais, sendo 72 machos e 79 fêmeas) que apresentavam catarata madura e hipermadura e baixa de visão utilizando Sistema Eletrorretinográfico BPM 200 (Retinographics Inc), na Clínica Veterinária Vetmasters, em São Paulo (SP), no período de setembro de 2004 a fevereiro de 2009, após terem sido submetidos ao exame oftalmológico que incluía teste de lágrima de Schirmer (Ophthalmos), teste de tingimento pela fluoresceína (Ophthalmos), tonometria de aplanação (Tonopen) e biomicroscopia com lâmpada de fenda (SL 15 Kowa).

Os animais foram separados em quatro grupos de acordo com a faixa etária: GI-1 a três anos e 11 meses (10 olhos), GII- quatro a sete anos e 11 meses de idade (86 olhos), GIII- oito a 11 anos e 11 meses de idade (115 olhos) e GIV- 12 anos ou mais (46 olhos). Todos os cães foram submetidos ao mesmo protocolo de sedação, e o exame foi realizado em sala escura. Com intuito de obter midríase, instilou-se colírio de tropicamida 1\%, em ambos os olhos. A adaptação ao escuro durou 30 minutos e foi feita com bandagem oclusiva. Para a sedação, administrou-se atropina $\left(0,044 \mathrm{mg} \mathrm{kg}^{-1}\right)$, por via subcutânea, e xilazina $(0,3 \mathrm{mg}$ $\left.\mathrm{kg}^{-1}\right)$ e butorfanol $\left(0,2 \mathrm{mg} \mathrm{kg}^{-1}\right)$, por via intramuscular.

Para a obtenção dos registros, utilizou-se eletrodo monopolar ERG-jet, que foi colocado sobre a córnea após preenchimento com metilcelulose $2 \%$ (Ophthalmos) com objetivo de proporcionar melhor contato elétrico e proteger a superfície corneal. O eletrodo de referência de cúpula de ouro (Grass) preenchido com creme eletrolítico foi colocado no canto temporal do olho, após tricotomia e higienização da pele. O eletrodo-terra, similar ao de referência, preenchido também com creme eletrolítico, foi colocado na face interna do pavilhão auricular previamente tricotomizado (Figura 1). Com os eletrodos devidamente colocados, posicionou-se o animal em decúbito lateral esquerdo para a realização da fase escotópica (escuro) no olho direito e depois decúbito lateral direito para a realização no olho esquerdo.

Para a resposta de bastonetes, utilizou-se, como estímulo, luz branca com sua intensidade máxima atenuada em duas unidades logarítimicas com filtro de densidade neutra. O intervalo entre os estímulos nessa fase foi de dois segundos. A segunda resposta, escotópica (claro), máxima, foi obtida com estímulos de alta intensidade, com intervalo de 10 segundos. Após adaptação ao claro de 10 minutos, registrou-se a resposta de cones, com luminância de aproximadamente $30 \mathrm{~cd} \mathrm{~m}^{2} \mathrm{e}$ intervalo entre os estímulos de um segundo. 


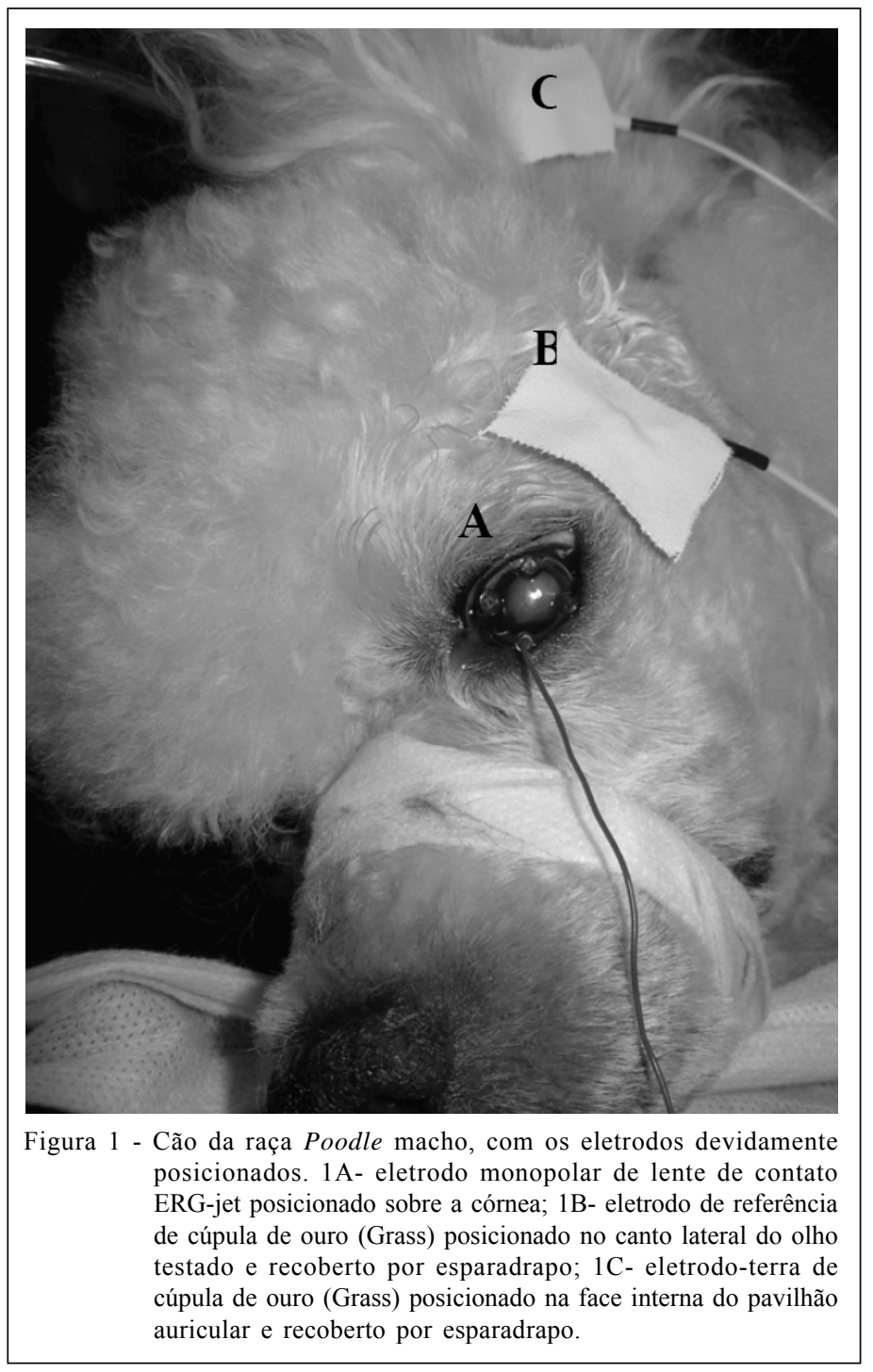

Para as três respostas, foram utilizados, em média, 16 estímulos, e a duração dos exames foi de aproximadamente 50 minutos, e 30 corresponderam ao tempo de adaptação ao escuro. Ao término do exame, mensurou-se a amplitude pico a pico (pico da onda-a até o pico da onda-b) em microvolts $(\mu \mathrm{V})$, e o tempo de culminação da onda-b (início do estímulo até o pico da onda-b) em milisegundos (ms), nas três respostas de cada paciente. Respostas atenuadas ou extintas foram excluídas da análise estatística. Caso o animal apresentasse resultado sugestivo de degeneração retiniana unilateral, manteve-se a resposta do melhor olho.

As respostas entre os diferentes grupos foram comparadas por meio da análise de variância (ANOVA), seguida de teste de Tukey-Kramer de múltiplas comparações. Quando as variáveis não apresentavam distribuição normal, foi realizado teste de Kruskal-Wallis (Anova não paramétrica), seguido de teste de Dunn de múltiplas comparações.

\section{RESULTADOS}

Os resultados obtidos da mensuração da amplitude pico a pico $(\mu \mathrm{V})$ e do tempo de culminação da onda-b (ms) da resposta de bastonetes, máxima resposta e resposta de cones nos quatro grupos subdivididos de acordo com a faixa etária estão apresentados na tabela 1 . Mensuraram-se valores mínimos, máximos, média, mediana e desvio padrão dentro de cada resposta.

Observou-se diminuição da amplitude quando se comparou GI com os demais grupos (Figura 2). Na resposta de bastonetes, essa diminuição foi significativa quando se comparou o GI com GIII e GIV. Na máxima resposta, essa diminuição foi significativa quando se comparou o GI com GIII e GIV, GII com GIV e GIII com GIV. Na resposta de cones, essa diminuição foi significativa quando se comparou GI com GIV, GII com GIV e GIII com GIV. Quanto ao tempo de culminação da onda-b, observou-se aumento quando foi comparado GI com os demais grupos, estatisticamente significante quando se comparou GI com GIII e GIV; e GII com GIV na resposta de bastonetes. Na máxima resposta e na resposta de cones, esse aumento não foi estatisticamente significativo.

\section{DISCUSSÃO}

O Eletrorretinograma de Campo Total é o teste eletrofisiológico mais utilizado na medicina veterinária por ser eficaz no auxilio diagnóstico de doenças que acometem as camadas mais externas da retina, sendo objetivo e detectando lesões retinianas antes mesmo das alterações fundoscópicas. A presença da catarata nos seus estágios mais avançados impossibilita a fundoscopia e por isso torna-se indispensável a utilização de outros meios de diagnóstico para certificar-se da funcionalidade retiniana. Nos cães, várias raças, dentre elas o Poodle, são geneticamente predispostas a serem portadoras de catarata e atrofia progressiva da retina (ADKINS \& HENDRIX, 2005). Assim sendo, o ERG nesses animais 
Tabela 1 - Amplitude ( $\mu \mathrm{V})$ e tempo de culminação (ms) das respostas de bastonetes, máximas respostas e respostas de cones obtidas nos quatro grupos de cães da raça Poodle portadores de catarata matura ou hipermadura e separados de acordo com a faixa etária.

\begin{tabular}{|c|c|c|c|c|c|}
\hline & Idade (anos) & 1 a 3 & 4 a 7 & 8 a 11 & 12 ou mais \\
\hline Grupo & & GI & GII & GIII & GIV \\
\hline Número (olhos) & & 10 & 86 & 115 & 46 \\
\hline \multirow{5}{*}{ Bastonetes - Amplitude $(\mu \mathrm{V})$} & Média & 100,87 & 75,22 & $60,75 * \dagger$ & $50,99 * \dagger$ \\
\hline & DP & 26,03 & 35,81 & 30,09 & 24,99 \\
\hline & Mediana & 103,56 & 66,59 & 57,78 & 50,28 \\
\hline & Mínimo & 60,42 & 27 & 16,53 & 11,25 \\
\hline & Máximo & 129,58 & 218,47 & 161,25 & 133,33 \\
\hline \multirow{5}{*}{$\begin{array}{l}\text { Bastonet es- Tempo de } \\
\text { culminação (ms) }\end{array}$} & Média & 54,50 & 58,56 & $60,77 *$ & $61,98 * \dagger$ \\
\hline & $\mathrm{DP}$ & 3,97 & 7,51 & 6,29 & 6,05 \\
\hline & Mediana & 53,5 & 58,47 & 61 & 60,75 \\
\hline & Mínimo & 50 & 36,8 & 35,75 & 50,75 \\
\hline & Máximo & 61,50 & 76,5 & 77 & 74,5 \\
\hline \multirow{5}{*}{$\begin{array}{l}\text { Máxima - Amplitude } \\
(\mu \mathrm{V})\end{array}$} & Média & 152,93 & 145,42 & $128,16 \dagger$ & $105,70 * \dagger+$ \\
\hline & DP & 32,05 & 40,95 & 46,81 & 44,22 \\
\hline & Mediana & 150,275 & 144,37 & 116,97 & 99,235 \\
\hline & Mínimo & 83,61 & 68,4 & 52,78 & 18,75 \\
\hline & Máximo & 200,03 & 275,56 & 295 & 208,89 \\
\hline \multirow{5}{*}{$\begin{array}{l}\text { Máxima - Tempo de culminação } \\
(\mathrm{ms})\end{array}$} & Média & 28,05 & 30,66 & 30,29 & 30,43 \\
\hline & $\mathrm{DP}$ & 1,28 & 4,20 & 2,87 & 2,79 \\
\hline & Mediana & 29 & 30 & 30 & 29,75 \\
\hline & Mínimo & 26,75 & 23 & 25 & 26,5 \\
\hline & Máximo & 31 & 47 & 41 & 39 \\
\hline \multirow{5}{*}{ Cones - Amplitude $(\mu \mathrm{V})$} & Média & 38,05 & 37,06 & 34,25 & $25,29 * \dagger+$ \\
\hline & $\mathrm{DP}$ & 8,93 & 16,68 & 19,79 & 16,81 \\
\hline & Mediana & 38,405 & 33,345 & 31,2 & 24,24 \\
\hline & Mínimo & 25,24 & 10,5 & 8,89 & 4,17 \\
\hline & Máximo & 48,33 & 86,7 & 121,94 & 105,1 \\
\hline \multirow{5}{*}{$\begin{array}{l}\text { Cones - Tempo de culminação } \\
(\mathrm{ms})\end{array}$} & Média & 25,33 & 26,82 & 26,99 & 25,31 \\
\hline & DP & 1,66 & 4,08 & 5,04 & 3,87 \\
\hline & Mediana & 25,87 & 26,5 & 26,5 & 25,5 \\
\hline & Mínimo & 21,5 & 16,5 & 7,3 & 16,25 \\
\hline & Máximo & 27,5 & 40,0 & 45 & 36 \\
\hline
\end{tabular}

$\mu \mathrm{V}=$ microvolts; ms=milisegundos; $\mathrm{DP}$ desvio padrão ; *: $\mathrm{P}<0,05$ diferente de um a três anos; $\dagger: \mathrm{P}<0,05$ diferente de quatro a sete anos; $+: \mathrm{P}<0,05$ diferente de oito a onze anos.

torna-se indispensável quando não se é capaz de ver o fundo do olho.

A interpretação do ERG deve ser feita levando-se em consideração a espécie, raça e idade do animal, além da opacidade de meios, do tipo de eletrodo, do eletrorretinógrafo e do protocolo de sedação (MENTZER et al., 2006). NARFSTRÖM et al. (2002) afirmam que não é possível comparar ERG de raças distintas, por apresentarem diferenças anatômicas, mesmo empregando um único protocolo anestésico.
Neste estudo, foram selecionados somente cães da raça Poodle em virtude da alta incidência de catarata nessa raça no nosso meio. Sabendo-se que a opacidade de meios atenua a resposta eletrorretinográfica, selecionaram-se cães com catarata no mesmo estágio de maturação.

A assimetria do número de cães com até três anos de idade, em comparação com os outros três grupos, se deu em virtude da catarata madura ou hipermadura acometer principalmente cães acima de 

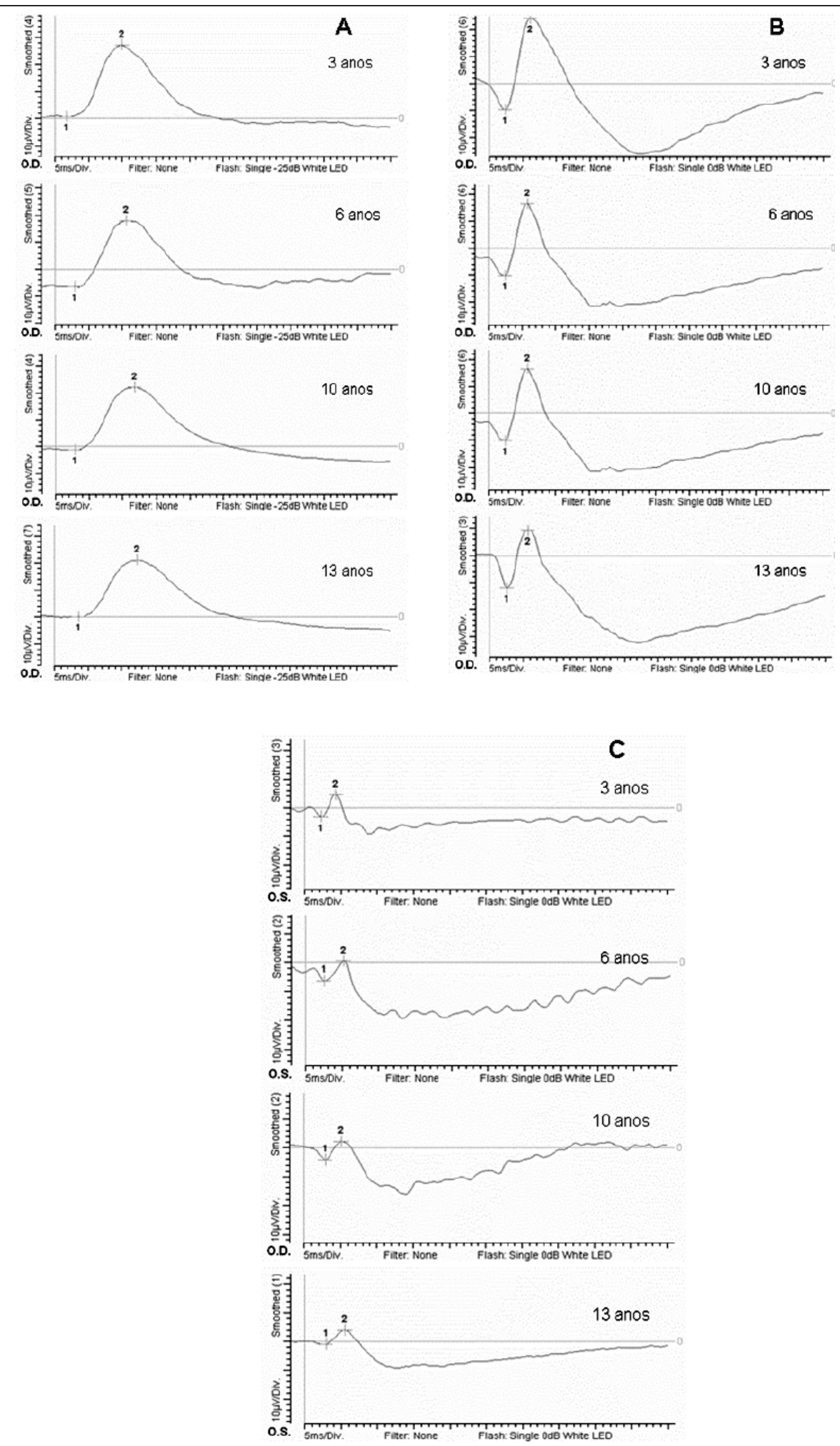

Figura 2 - Traçados eletrorretinográficos dos cães da raça Poodle portadores de catarata madura ou hipermadura em que são observadas as respostas nos quatro grupos subdivididos de acordo com a faixa etária. 2A- Resposta de Bastonetes, 2B- Máxima Resposta e 2CResposta de Cones. Observar a diminuição gradativa da amplitude das respostas de acordo com o envelhecimento do animal. 
quatro anos. Catarata em estágios iniciais acomete Poodles jovens, mas estes foram excluídos deste estudo, pois foram selecionados cães com catarata madura ou hipermadura, com intuito de padronizar os valores do ERG nessa raça com baixa de visão em razão da opacidade de meios (catarata).

Para a realização do exame, necessita-se da cooperação do paciente, que deve permanecer imóvel. O protocolo de sedação empregado em todos os animais deste trabalho, utilizando associação de atropina, xilazina e butorfanol, permitiu registros adequados, com baixo ruído. Dependendo da escolha do anestésico, a alteração do ERG pode ser mínima ou importante quando utilizados barbitúricos ou anestésicos voláteis (NARFSTRÖM et al., 2002; NORMAN et al., 2008).

Sabe-se que as respostas podem ser variadas de acordo com o eletrodo escolhido. Eletrodos corneais, tais como ERG-jet, Burian Allen e Goldlens, são capazes de obter respostas reprodutíveis e estáveis, pois a superfície de registro está sempre posicionada no mesmo lugar, ou seja, na córnea, onde se obtêm a máxima voltagem, porém podem causar lesão corneal, limitando o tempo do exame (HÉRBERT et al., 1996). Eletrodos alternativos menos invasivos, como DTL (Dawson Trick Litzkov), que é feito de microfibra, pode ser usado nos animais, mas é mais difícil que no homem devido à inadequada fixação nos cantos medial e lateral pela presença de pelos (MIERDEL, 1995). Esse eletrodo, por ser mais confortável, pode ser utilizado em exames demorados, não causando qualquer tipo de lesão ocular (HÉRBERT et al., 1996). Outro tipo de eletrodo corneal acoplado a uma fonte luminosa foi utilizado com sucesso em cães da raça Beagle, porém foram necessários dois tamanhos diferentes (MAEHARA et al., 2005).

O eletrodo descartável monopolar ERG-jet foi eleito neste estudo, por este ser acessível e bem tolerado por todos os animais, sendo capaz de registrar respostas com baixa interferência. O seu tamanho único permitiu perfeita colocação sobre a superfície corneal em todos os cães, independentemente da abertura da fenda palpebral, e não se observou lesão corneal ao término do exame. Acredita-se que a proteção com metilcelulose $2 \%$ acrescida de colírio anestésico foi indispensável para que não ocorresse tal dano.

O ideal seria que cada laboratório obtivesse valores de normalidade para cada espécie e cada raça, com pelo menos três grupos de idade (juvenil, adulto e idoso), pois, quanto mais jovem, maiores são as amplitudes e, quanto mais velho, menores são as amplitudes das respostas, semelhante ao que ocorre no ERG humano (NARFSTRÖM et al., 2002). Com esse intuito de padronizar os valores, separaram-se os animais em quatro grupos, e observou-se que, quanto mais idosos eram os cães, menores eram os valores da amplitude nas três respostas testadas. Observou-se também que o tempo de culminação da onda-b aumenta na resposta de bastonetes e se mantém praticamente inalterado nas outras duas respostas, confirmando que a diminuição fisiológica dos fotorreceptores nos pacientes idosos interfere principalmente na amplitude e não no tempo de culminação da onda-b.

\section{CONCLUSÕES}

A idade dos cães interferiu diretamente nos resultados do exame eletrorretinográfico. Os cães mais velhos, quando comparados com os mais jovens, apresentaram diminuição da amplitude na resposta de bastonetes, máxima resposta e resposta de cones. $\mathrm{O}$ aumento do tempo de culminação da onda-b foi observado na resposta dos bastonetes. Quando se avaliou a resposta dos cones, não se observou tal atraso e o tempo de culminação da onda-b foi semelhante nos quatro grupos testados. Dessa forma, conclui-se que, com o envelhecimento e a consequente perda fisiológica dos fotorreceptores, a mensuração da amplitude é mais significante que o tempo de culminação.

A padronização do eletrorretinograma, assim como dos parâmetros de normalidade de um laboratório de ERG, são indispensáveis quando se objetiva auxílio no diagnóstico das degenerações retinianas e se almeja comparar diversos exames, mesmo quando apresentam opacidade de meios. O estudo permitiu determinar, nos cães da raça Poodle portadores de catarata madura ou hipermadura, os parâmetros de normalidade, com os valores mínimos e máximos, em cada faixa etária para cada resposta testada.

\section{REFERÊNCIAS}

ADKINS, E.A.; HENDRIX, D.V.H. Outcomes of dogs presented for cataract evaluation: a retrospective study. Journal of the American Animal Hospital Association, v.41, p.235-240, 2005 .

BIRCH, D.G. Clinical electroretinography. Ophthalmology Clinics of North America, v.2, n.3, p.469-497, 1989.

BIRCH, D.G.; ANDERSON, M.S. Standardized full-field electroretinography. Archives of Ophthalmology, v.110, p.1571-1576, 1992.

DANTAS, A.M. Eletrorretinografia. In: DANTAS, A.M. et al. Eletrofisiologia ocular. Rio de Janeiro: Cultura Médica, 1995. p.141-225.

GELATT, K.N.; MACKAY, E.O. Prevalence of primary breedrelated cataracts in the dog in North America. Veterinary 
Ophthalmology, v.8, n.2, p.101-111, 2005. Disponível em: $<$ http://www3.interscience.wiley.com/cgi-bin/fulltext/ 118669412/PDFSTART>. Acesso em: 19 nov. 2009. doi: 10.1111/j.1463-5224.2005.00352.x.

GONÇALVES, E.R. et al. Eletrofisiologia ocular. In: CONGRESSO BRASILEIRO DE OFTALMOLOGIA SOCIEDADE BRASILEIRA DE RETINA E VÍTREO. RETINA E VÍTREO: CLÍNICA E CIRURGIA, 2000, São Paulo, SP. Anais... São Paulo: Roca, 2000. p.185-225.

HÉRBERT, M. et al. Reproducibility of electroretinograms recorded with DTL electrodes. Documenta Ophthalmologica, v.91, p.333-342, 1996. Disponível em: <http:// www.springerlink.com/content/n 17902333 t2 x 0 q tk $? p=4910575 b e 6354 \mathrm{c} 5 \mathrm{cb} 2 \mathrm{~b} 7 \mathrm{bacfa} 2224 \mathrm{~b} 2 \mathrm{~d} \& \mathrm{pi}=6>$. Acesso em: 19 nov. 2009. doi: 10.1007/BF01214651.

KOMAROMY, A.M. et al. Electroretinography in dogs and cats. Part I. Retinal morphology and physiology. Compendium on Continuing Education for the Practicing Veterinarian, v.20, p.343-354, 1998a.

KOMAROMY, A.M. et al. Eletroretinography in dogs and cats Part II. Technique, interpretation and indications. Compendium on Continuing Education for the Practicing Veterinarian, v.20, p.355-366, 1998 b.

MAEHARA, S. et al. Electroretinography using contact lens electrode with built-in light source in dogs. Journal of Veterinary Medical Science, v.67, n.5, p.509-514, 2005. Disponível em: <http://www.jstage.jst.go.jp/article/jvms/67/5/ 509/ pdf>. Acesso em: 19 nov. 2009. doi: 10.1292/jvms.67509 JOI JSTTAGE/jvms/67.509.

MARMOR, M.F.; ZRENNER, E. Standard for clinical electrretinography (1999 Update). Documenta Ophthalmologica, v.97, p.143-156, 1998. Disponível em: http://www.springerlink.com/content/w2t56570w3505672/ $? \mathrm{p}=88 \mathrm{dc} 35 \mathrm{~d} 2212646059 \mathrm{~d} 7 \mathrm{da} 807401 \mathrm{e} 624 \mathrm{~d} \& \mathrm{pi}=3$. Acesso em: 19 nov. 2009. doi : 10.1023/A:1002016531591.

MENTZER, A.L. et al. Influence of recording electrode type and reference electrode posicion the canine electroretinogram. Documenta Ophtalmologica,v.111, n.2, p.95-106, 2006. Disponível em: <http:// www.springerlink.com/content/a 88071 r431801387/ $? \mathrm{p}=4 \mathrm{~d} 3 \mathrm{e} 730 \mathrm{ccd} 854 \mathrm{ec} 691195 \mathrm{fb} 03684 \mathrm{a} 657 \& \mathrm{pi}=4>$. Acesso em: 19 nov. 2009. doi: 10.1007/s10633-005-4517-7.

MIERDEL, P. An improved holder for the DTL fiber electrode in electroretinography. Documenta Ophthalmologica, v.89, p. 249-250, 1995. Disponível em: <http://www.springerlink.com/ c o $\mathrm{n} \mathrm{t} \mathrm{e} \mathrm{n} \mathrm{t} \mathrm{/} \mathrm{q} 2$ p 5 k $8 \begin{array}{llllllllll} & \text { q } & 5 & 4 & 5 & 2 & \text { v } & 0 & 1 & 5\end{array}$ $? \mathrm{p}=\mathrm{db} 46 \mathrm{~b} 6 \mathrm{e} 9 \mathrm{a} 18046 \mathrm{dbb} 592496828055924 \& \mathrm{pi}=5>$. Acesso em: 19 nov. 2009. doi: 10.1007/BF01203378.
NARSTRÖM, K. et al. Guidelines for clinical electroretinography in the dog. Documenta Ophtalmologica, v.105, p.83-92, 2002. Disponível em: <http:// www.springerlink.com/content/xnt2386411514h14/ $? \mathrm{p}=8750 \mathrm{~b} 67 \mathrm{dac} 0140 \mathrm{ad} 9331470 \mathrm{af9} 823219 \& \mathrm{pi}=0>$. Acesso em: 19 nov. 2009 . doi: 10.1023/A:1020524305726

NARFSTRÖM, K. et al. Neuronal ceroid lipofuscinosis: clinical and morphologic findings in nine affected Polish Owczarek Nizinny (PON) dogs. Veterinary Ophthalmology, v.10, n.2, p.111-120, 2007. Disponível em: $<$ http://www3.interscience.wiley.com/ cgi-bin/fulltext/118507731/PDFSTART $>$. Acesso em: 19 nov. 2009 . doi: $10.1111 / \mathrm{j} .1463$ $5224.2007 .00527 . x$.

NORMAN, J. et al. The effects of medetomidine hydrochloride on the electroretinogram of normal dogs. Veterinary Ophthalmology, v.11, n.5, p.299-305, 2008. Disponível em: <http://www3.interscience.wiley.com/cgi-bin/fulltext/ 121382044/PDFSTART>. Acesso em: 19 nov. 2009. doi: 10.1111/j.1463-5224.2008.00650.x.

OFRI, R. et al. Chronic ocular hypertension alters local retinal responsivenes. British Journal of Ophthalmology, v.77, p.502-508, 1993. Disponível em: <http://bjo.bmj.com/content/ $77 / 8 / 502$.ful1.pdf?sid=4 a 788 d f - 28 be -44 d 4 b117a0e18c4756de>. Acesso em: 19 nov. 2009. doi: 10.1136/ bjo.77.8.502.

PETERSEN-JONES, S. et al. Electroretinograms of dog and chicken. In: HECKENLIVELY, J.R.; ARDEN, G.B. Principles and practice of clinical electrophysiology of vision. 2.ed. Cambridge: The Mit, 2006. p.911-921.

RUBIN, L. F. Clinical features of hemeralopia in adult Alaskan Malamute. Journal of American Veterinary Medical Association, v.158, n.10, p.1696-1698, 1971.

SAFATLE, A.M.V. et al. Retinal degeneration in a Pit Bull dog: electroretinographic findings. Archives of Veterinary Science, v.10, n.2, p.119-124, 2005.

SIMS, M.H. Electrodiagnostic evaluation of vision. In: GELATT K.N. Veterinary ophthalmology. 3.ed. Philadelphia: Lippincott Williams e Wilkins, 1999. p.483507.

TZECOV, R.; ARDEN, G.B. The electroretinogram in diabetic retinopathy. Survey of Ophthalmology, v.44, n.1, p.53-60, 1999.

YANASE, J.; OGAWA, H. Effects of halothane and sevoflurane on the electroretinogram of dogs. American Journal of Veterinary Research, v.58, n.8, p.904-909, 1997. 